\title{
Alternative descriptors of Coulomb systems and their relationship to the kinetic energy
}

\author{
Á. Nagy \\ Department of Theoretical Physics, University of Debrecen, \\ H-4010 Debrecen, Hungary
}

(May 20, 2008)

\begin{abstract}
A family of quantities capable of fully determining every property of a Coulomb system is defined. Making use of the differential virial theorem of Nagy and March, a first-order differential equation for the functional derivative of the kinetic energy functional is derived for spherically symmetric systems.
\end{abstract}




\section{INTRODUCTION}

The ground-state electron density is a fundamental quantity. It determines every property of the electron system [1]. Recently, other quantities have been turned out to be descriptors of a Coulomb system. The shape function, or density per particle was the first quantity, that has been shown [2] to provide a full description of a Coulomb system. According to a recent paper [3] such reactivity indicators as Fukui function, local softness, softness kernel and the electrostatic potential are all capable of fully determining every property of a Coulomb system. It has recently been presented [4] that local kinetic energy and local temperature are also adequate for describing a Coulomb system. Here, a broader family of descriptors of Coulomb systems is presented.

Nowadays, density functional calculations are mainly based on the Kohn-Sham scheme. There is, however, a growing interest in orbital-free calculations, too. These are inexpensive, but inaccurate. The main problem is the lack of accurate approximation for the kinetic energy functional. Making use of the differential virial theorem of Nagy and March [6], first-order differential equation for the functional derivative of the kinetic energy is derived for spherically symmetric systems.

\section{NEW DESCRIPTORS OF COULOMB SYSTEMS}

We start out from the Kohn-Sham equations [7]

$$
-\frac{1}{2} \nabla^{2} u_{k}+v_{\mathrm{KS}} u_{k}=\epsilon_{k} u_{k}
$$

where $v_{\mathrm{KS}}, u_{k}$ and $\epsilon_{k}$ are the Kohn-Sham potential, the orbitals and the orbital energies, respectively. Consider the expression

$$
\xi(\mathbf{r})=\sum_{k}^{N} \alpha_{k} n_{k},
$$

where $\alpha_{k} \geq 0$ for $1 \leq k<N$ and $\alpha_{N}>0$.

$$
n_{k}=\left|u_{k}\right|^{2}
$$


are the one-partical densities. The sum is going for the occupied orbitals and $N$ is the number of electrons.

Theorem 1. For any Coulomb system, $\xi$ determines the external potential $v$ up to an additive costant. $\xi$ also determines the number of electron.

Proof: The external potential of a Coulomb system has the form

$$
v(\mathbf{r})=-\sum_{\beta} \frac{Z_{\beta}}{\left|\mathbf{r}-\mathbf{R}_{\beta}\right|},
$$

where $Z_{\beta}$ is the atomic number of the nucleus at the position $\mathbf{R}_{\beta}$. Now, we show that the atomic numbers can be derived from the cusp condition for $\xi$. The cusp relations for orbitals are known from earlier studies [8-14] and we can immediatelly obtain the corresponding relations for $\xi$. Expanding the orbital $u_{k}$ around the nucleus $\beta$

$$
u_{k}=\sum_{l m} a_{k l m} r^{l} \chi_{k l m}(r) Y_{l m}(\hat{r}),
$$

where $Y_{l m}$ are the spherical harmonics, the cusp relation for the radial function $\chi_{k l m}$ is $[11-13]$

$$
\chi_{k l m}^{\prime}\left(\mathbf{R}_{\beta}\right)=-\frac{Z}{l+1} \chi_{k l m}\left(\mathbf{R}_{\beta}\right)
$$

From Eqs. (3), (5) and (6) the relation for spherical average of the orbital density $n_{k}$ can be obtained. Define the function $\gamma_{k l}(r)$ as

$$
\gamma_{k l}(r)=\frac{\bar{n}_{k}(r)}{r^{2 l}}
$$

where $l$ is the smallest integer for which $\chi_{k l m}$ is not zero at the nucleus $\beta$ [11-13]. Eqs. (3), (5), (6) and (7) lead to the relation

$$
\gamma_{k l}^{\prime}(r)\left(\mathbf{R}_{\beta}\right)=-2 \frac{Z_{\beta}}{l+1} \gamma_{k l}\left(\mathbf{R}_{\beta}\right)
$$

If $l=0 \mathrm{Eq}$. (8) reduces to

$$
\bar{n}_{k}^{\prime}\left(\mathbf{R}_{\beta}\right)=-2 Z_{\beta} \bar{n}_{k}\left(\mathbf{R}_{\beta}\right)
$$

Turning to the new descriptor $\xi$ (Eq. (2)) and defining the function $\eta_{l}(r)$ 


$$
\eta_{l}(r)=\frac{\bar{\xi}(r)}{r^{2 l}}
$$

we arrive at the cusp relation

$$
\eta_{l}^{\prime}\left(\mathbf{R}_{\beta}\right)=-2 \frac{Z_{\beta}}{l+1} \eta_{l}\left(\mathbf{R}_{\beta}\right)
$$

If $l=0$, that is, the factor $\alpha$ is larger than zero for at least one of the $s$ orbitals, Eq. (11) reduces to

$$
\bar{\xi}^{\prime}\left(\mathbf{R}_{\beta}\right)=-2 Z_{\beta} \bar{\xi}\left(\mathbf{R}_{\beta}\right)
$$

The positions of cusps in $\xi$ locate the nuclei and Eq. (12) determine the atomic numbers.

To prove that $\xi$ determines also the number of electrons, we have to keep in mind that the asymptotic decay of $\xi$ is given by the highest occupied orbital [15-18]:

$$
\xi(r \rightarrow \infty) \sim n_{N}(r \rightarrow \infty) \sim r^{2\left(\frac{z_{t o t}-N+1}{\sqrt{2 I_{N}}}-1\right)} e^{-2 r \sqrt{2 I_{N}}},
$$

where $I_{N}$ is the vertical ionization potential of the $N$-electron system and $Z_{\text {tot }}=\sum_{\beta} Z_{\beta}$ is the sum of the atomic numbers. Following Ayers $[2,3]$ the number of electrons can be obtained from the polynomial part of Eq. (13):

$$
N=1+\left(Z_{t o t}+\frac{1}{4}\left[\left(\frac{\partial \ln \xi}{\partial r}\right)\left(\frac{\partial^{2} \ln \xi}{\partial r \partial\left(\frac{1}{r}\right)}+2\right)\right]_{r \rightarrow \infty}\right)
$$

\section{Examples:}

If $\alpha_{k}$ are the occupation numbers: $\alpha_{k}=\lambda_{k} \xi$ is the electron density.

If $\alpha_{k}=\lambda_{k} / N, \xi$ is the shape function $\sigma: \xi=\sigma=n / N$.

If $\alpha_{1}=\ldots=\alpha_{N-1}=0$ and $\alpha_{N}=1$, that is, $\xi=n_{H O M O} \approx f^{-}$is the highest occulied orbital density which approximates the Fukui function. This result is related to a previous work of Ayers [5] which showed that the frontier molecular orbitals (highest occupied and lowest unoccupied orbitals) suffice to determine the electron density, and thus all properties of a system (even if the system is not Coulombic). 
If only a single $\alpha_{k}$ is non-zero: $\alpha_{k}=1$ and $\alpha_{l}=0, l \neq k$, is the case studied in [4]. It was shown that a single Kohn-Sham orbital for a Coulomb system is a descriptor of a Coulomb system.

In the next section two new quantities $g(\mathbf{r})($ Eq. (29)) and $q(\mathbf{r})$ (Eq. (18)) are introduced that are examples of $\xi$ for special choice of $\alpha_{k}$. These new descriptors, together with the density appear in the first-order differential equation for the functional derivative of the kinetic energy.

\section{THE FUNCTIONAL DERIVATIVE OF THE KINETIC ENERGY FUNCTIONAL}

In spherically symmetric systems the Kohn-Sham equations take the form

$$
-\frac{1}{2} \frac{d^{2} P_{k}}{d r^{2}}+\frac{l_{k}\left(l_{k}+1\right)}{2 r^{2}} P_{k}+v_{\mathrm{KS}} P_{k}=\epsilon_{k} P_{k}
$$

where $P_{k}=r R_{k}(r)$ are the radial wave functions.

The differential form of the virial theorem for spherically symmetric Kohn-Sham potential $v_{\mathrm{KS}}(r)[6]$ is

$$
\tau^{\prime}=-\frac{1}{8} \varrho^{\prime \prime \prime}-\frac{1}{2} \varrho v_{\mathrm{KS}}^{\prime}+\frac{\tilde{q}^{\prime}}{r^{2}}-\frac{\tilde{q}}{r^{3}}
$$

where

$$
\tilde{q}=4 r^{2} \pi q
$$

and

$$
q=\frac{1}{2} \sum_{k} \lambda_{k} l_{k}\left(l_{k}+1\right) n_{k} .
$$

The radial density and the radial kinetic energy density are given by

$$
\varrho=4 r^{2} \pi n
$$

and 


$$
\tau(r)=-\left.\frac{1}{2} \frac{\partial^{2} \varrho\left(r^{\prime}, r\right)}{\partial r^{2}}\right|_{r^{\prime}=r}+\frac{1}{2} \sum_{k} \lambda_{k} l_{k}\left(l_{k}+1\right) \frac{\varrho_{k}(r)}{r^{2}}
$$

respectively.

$$
\varrho_{k}=P_{k}^{2}
$$

are the one-partical radial densities. (For closed shells the functions $q$ and $g$ are spherically symmetric.)

For particles having zero angular momentum i.e. for $s$ electrons the differential virial theorem reduces to the special form of March and Young [19]

$$
\tau^{\prime}=-\frac{1}{8} \varrho^{\prime \prime \prime}-\frac{1}{2} \varrho v_{\mathrm{KS}}^{\prime}
$$

The Euler equation of the density functional can be written as

$$
\frac{\delta T_{s}}{\delta n}+v_{\mathrm{KS}}=\mu
$$

where $\mu$ is the chemical potential [20-22].

The radial kinetic energy density can also be written as

$$
\tau(r)=4 r^{2} \pi t(r)
$$

where

$$
t(r)=-\frac{1}{2} \sum_{k} \lambda_{k} u_{k}^{*} \nabla^{2} u_{k}
$$

Combining Eqs. (1), (23) and (25) we arrive at the relation:

$$
t(r)=n \frac{\delta T_{s}}{\delta n}+\sum_{k}\left(\epsilon_{k}-\mu\right) n_{k}
$$

From Eqs. (24) and (26) we are led to the relation

$$
\tau=\varrho \frac{\delta T_{s}}{\delta n}-\mu \varrho+\tilde{g},
$$

where 


$$
\tilde{g}=4 r^{2} \pi g(r)
$$

and

$$
g(r)=\sum_{k} \lambda_{k} \epsilon_{k} n_{k}
$$

Differentiating Eq. (27) with respect to $r$ and substituting it into the differential virial theorem (16) we obtain

$$
\frac{1}{2} \varrho\left(\frac{\delta T_{s}}{\delta n}\right)^{\prime}+\varrho^{\prime} \frac{\delta T_{s}}{\delta n}=\tilde{f},
$$

where

$$
\tilde{f}=-\frac{1}{8} \varrho^{\prime \prime \prime}-\tilde{g}^{\prime}+\mu \varrho^{\prime}+\frac{\tilde{q}^{\prime}}{r^{2}}-\frac{\tilde{q}}{r^{3}} .
$$

The total non-interacting kinetic energy can be separated as

$$
T_{s}=T_{w}+T_{p}
$$

where the $T_{w}$ is the full Weizsäcker kinetic energy [23]

$$
T_{w}=\frac{1}{8} \frac{|\nabla n|^{2}}{n}
$$

and the term $T_{p}$ is called Pauli energy [24-26] The functional derivatives are

$$
\frac{\delta T_{w}}{\delta n}=\frac{1}{8}\left|\frac{\nabla n}{n}\right|^{2}-\frac{1}{4} \frac{\nabla^{2} n}{n}=n^{-1 / 2}\left(-\frac{1}{2} \nabla^{2}\right) n^{1 / 2}
$$

and

$$
v_{p}=\frac{\delta T_{p}}{\delta n}
$$

Substituting Eqs. (32)-(35) into Eq. (30) we arrive at a first-order differential equation for the functional derivative of the Pauli energy, that is, for the Pauli potential $v_{p}$ :

$$
\frac{1}{2} \varrho\left(\frac{\delta T_{p}}{\delta n}\right)^{\prime}+\varrho^{\prime} \frac{\delta T_{p}}{\delta n}=f
$$

where 


$$
f=-\tilde{g}^{\prime}+\mu \varrho^{\prime}+\frac{\tilde{q}^{\prime}}{r^{2}}-\frac{\tilde{q}}{r^{3}}
$$

In the knowledge of the functions $\varrho(r), g(r)$ and $q(r)$ the differential equation (37) can be solved and the Pauli potential can be written as

$$
v_{p}=\frac{2}{\varrho^{2}} \int_{\infty}^{r} \varrho\left(r_{1}\right) f\left(r_{1}\right) d r_{1} .
$$

Eq. (38) is the main result of this paper. With Eq. (34) it gives the functional derivative of the kinetic energy. Then the kinetic energy density $\tau$ can be obtained from Eq. (27) and the integration of $\tau$ leads to the total kinetic energy.

\section{ILLUSTRATIVE EXAMPLES}

The simplest examples are, of course, H-atom, H-like ions and He-atom, He-like ions. In these trivial cases, the total kinetic energy is the Weizsäcker kinetic energy and the Pauli energy and potential are zero. Eq. (37) gives $f=0$, because $\tilde{g}=\mu \varrho$ and $q=0$.

As a non-trivial example consider the Be atom and isoelectronic ions. In this case $q=0$. It is instructive to introduce the function $\theta$ with the Dawson-March transformation [27]:

$$
\begin{aligned}
& P_{1}=\frac{1}{\sqrt{\lambda_{1}}} \varrho^{1 / 2} \cos \theta \\
& P_{2}=\frac{1}{\sqrt{\lambda_{2}}} \varrho^{1 / 2} \sin \theta
\end{aligned}
$$

Eqs. (39) and (40) lead to the relation between the radial electron density and the phase $\theta$ :

$$
\theta^{\prime \prime}+\frac{\varrho^{\prime}}{\varrho} \theta^{\prime}=2 \zeta \sin (2 \theta)
$$

where

$$
\zeta=\frac{1}{2}\left(\epsilon_{1}-\epsilon_{2}\right)
$$

After some mathematics Eq. (38) gives

$$
v_{p}=\frac{1}{2}\left(\theta^{\prime}\right)^{2}-2 \zeta \cos ^{2} \theta
$$

In the knowledge of the density, the differential equation (41) can be numerically solved and the relation (43) gives the Pauli potential. 


\section{DISCUSSION}

In the ground state the density not only determines every property of an electronic system but there also exists a variational principle [1]. The quantities $\xi$ do not obey a variational principle and are descriptors of a Coulomb system only. This is an important limitation. Still they can be used in a large number of systems (atoms, molecules, etc.).

We would like to mention that the local ionization potential $\tilde{\varepsilon}(\mathbf{r})=\sum_{k} \varepsilon_{k} n_{k}(\mathbf{r}) / n(\mathbf{r})$ introduced by Politzer et al. $[28,29]$ is closely related to the function $g: \tilde{\varepsilon}(\mathbf{r}) n(\mathbf{r})=g(\mathbf{r})$. The local ionization potential is a measure of chemical reactivity and is linked to the local temperature, and thus to the local kinetic energy [30]. So there is a very intimite relationship between the function $g$ and the local kinetic energy $t$. A further study of this relationship can help finding accurate approximation for the function $g$ and would point toward practical applications in orbital-free DFT.

In the differential equation (36) derived for the Pauli potential the knowledge of the functions $\varrho, q$ and $g$ are needed to obtain a solution. Of course, in principle one of them is enough, as the other two quantities can be given as a functional of the selected descriptor. To find relations between them will be the subject of further studies.

Finally, we sketch how orbital-free solutions could be obtained based on the results of this paper provided that the functions $q$ and $g$ can be accurately approximated with a functional of $\varrho$.

(i) Starting from an initial "guessed" electron density $\varrho$, calculate the functions $q$ and $g$ and $\tilde{q}$ and $\tilde{g}$ using Eqs. (17), (18), (28) and (29).

(ii) Using Eq. (37) calculate the function $f$. Note that the asymptotic decay of the density determines the chemical potential $\mu$.

(iii) Solve Eq. (36) for the Pauli potential $v_{p}$ using Eq. (38).

(iv) Solve the Euler equation (23) to obtain the electron density $\varrho$. Note that one needs the Kohn-Sham potential to obtain the solution. The accuracy of the results will depend on the approximate exchange-correlation functional used in solving the Euler equation. 
(v) Repeat steps (i)-(iv) until convergence. Then calculate the total non-interacting kinetic energy from Eq. (27).

Whether this algorithm provides an efficient and accurate way to solve the Euler equation depends on how accurately the functions $q$ and $g$ are approximated using the density. It might turn out that it is more appropriate to use the descriptor $q$ or $g$ instead of the density. 


\section{REFERENCES}

[1] P. Hohenberg and W. Kohn, Phys. Rev. 136, B864 (1864).

[2] P. W. Ayers, Proc. Natl. Acad. Sci.97, 1959 (2000).

[3] P. W. Ayers, Chem. Phys. Lett.438, 148 (2007).

[4] P. W. Ayers and Á. Nagy, J. Chem. Phys. 126, 144108 (2007).

[5] P. W. Ayers, Theor. Chem. Acc. 110, 267 (2003).

[6] Á. Nagy and N. H. March, Phys. Rev. A40, 554 (1989).

[7] W. Kohn and L. J. Sham,Phys. Rev. 140, A1133 (1865).

[8] T. Kato, Commun. Pure Appl. Math. 10, 151 (1957). E.

[9] Steiner, J. Chem. Phys. 39, 2365 (1963).

[10] R. T. Pack and W. B. Brown, J. Chem. Phys. 45 , 556 (1966).

[11] Á Nagy and K. D. Sen, J. Phys. B 33, 1745 (2000).

[12] Á Nagy and K. D. Sen, Chem. Phys. Lett. 332, 154 (2000).

[13] Á Nagy and K. D. Sen, J. Chem. Phys. 115, 6300 (2001).

[14] N. H. March, I. A. Howard, A. Holas, P. Senet and V. E. Van Doren, Phys. Rev. A 63, $012520(2001)$.

[15] R. Ahlrichs, J. Chem. Phys. 64, 2706 (1976).

[16] M. Hoffmann-Ostenhof and T. Hoffmann-Ostenhof, Phys. Rev. A 16, 1782 (1977).

[17] H. J. Silverstone, Phys. Rev. A 23, 1030 (1981).

[18] M. Levy, J. P. Perdew and V. Sahni, Phys. Rev. A 30, 2745 (1984).

[19] N. H. March and W. H. Young, Nucl. Phys. 12, 237 (1959). 
[20] I. Lindgren and S. Salomonson, Phys. Rev. A 67, 056501(2003).

[21] R. van Leeuwen, Adv. Quantum Chem. 43, 24 (2003).

[22] S. B. Liu and P.W. Ayers, Phys. Rev. A 70, 022501 (2004).

[23] C. F. Weizsäcker, Z. Phys. 96341 (1935).

[24] N. H. March, Phys. Lett. A 113, 476 (1986).

[25] M. Levy, J. P. Perdew, and V. Sahni, Phys. Rev. A 30, 2745 (1984).

[26] S. B. Liu, J. Chem. Phys. 126, 244103 (2007).

[27] K. A. Dawson and N. H. March, J. Chem. Phys. 81, 5850 (1984).

[28] P. Politzer, J. S. Murray, M. E. Grice, T. Brinck and S. Ranganathan, J. Chem. Phys. 95, 6699 (1991).

[29] J. S. Murray, J. M. Seminario, P. Politzer and P. Sjoberg, Int. J. Quantum Chem. 24, 645 (1990).

[30] P. W. Ayers, R. G. Parr and Á. Nagy, Int. J. Quantum Chem. 90, 309 (2002).

\section{ACKNOWLEDGEMENTS}

This work was supported by the grant OTKA No. T 67923. 